\title{
Cosmic Ray production in SNRs of Pulsar Wind Nebulae type at different ages
}

\author{
Vera G. Sinitsyna ${ }^{1, *}$ and Vera Y. Sinitsyna ${ }^{1}$ \\ ${ }^{1}$ P.N. Lebedev Physical Institute, Moscow, Russia
}

\begin{abstract}
The overall observations of plerions from radio to the very high energy gamma-rays could provide information about the evolution of PWN from the young Crab-like to the older stages. The extended $\mathrm{MeV}-\mathrm{TeV}$ emission from Geminga middle-aged pulsar of $\sim 3.4 \times 10^{5}$ year age in SHALON, Milagro, HAWC observations, and Fermi-LAT detection could arise from the PWN associated with the Geminga SNR. The TeV $\gamma$-ray emission from 3C $58 \mathrm{PWN}$ whose estimated age varies from $\sim 800$ to $(5-7) \times 10^{3}$ years was first detected in year 2011 by SHALON. The overall spectral energy distribution and information about the extension of PWN from radio to GeV-TeV energies from Fermi-LAT and SHALON observations can contribute to particle transport models and also to the understanding of the mechanisms of PWN expanding, which is, in turn, can shed light on the age of 3C 58 and the history of progenitor SN explosion.
\end{abstract}

\section{Introduction}

Pulsar wind nebulae (PWNe) are powerful galactic sources of very high energy gamma-rays. PWNe are created by the interaction of pulsar winds with the surrounding media. Such objects contain a pulsar left over the supernova explosion having large electric and magnetic fields and generating a pulsar wind, the outflow of pulsar-accelerated particles [1]. Then the interaction of pulsar winds with the surrounding media generating a shock region where particles are accelerated (e.g. [2]). PWN observations in the wide energy range show that the photon spectra vary with radial distance in some of these objects. So, such observations including ones at high energies can provide information on the energy-dependent and spatially-dependent mechanisms of particle acceleration within the nebula.

\section{Geminga}

A neutron star in the constellation Gemini is the second brightest source of $\mathrm{MeV}-\mathrm{GeV} \gamma$-rays in the sky, discovered in 1972 by SAS-2 which had no counterpart in other wavelengths. Only in 1991, in X-ray observations by ROSAT satellite, a regular periodicity of 0.237 seconds was detected. This makes Geminga the closest known pulsar to Earth. Geminga is suggested to be a supernova remnant with the expected age of $\sim 3.4 \times 10^{5} \mathrm{yr}[3,4]$. Sweeping of the interstellar medium could shape the pulsar wind nebula around Geminga. The morphology of the PWN is unusual, as there is no obvious bow-shock at the front of the nebula, although there is a candidate shock around the pulsar in the optical [5]. The nebula has been extensively studied in X-rays. Also, the very high energy gamma-rays from Geminga [6, 7] were detected by SHALON experiment at $0.8-6 \mathrm{TeV}$ (Fig. 1). This weak TeV-emission coincides with the

\footnotetext{
*e-mail: sinits@sci.lebedev.ru
} 

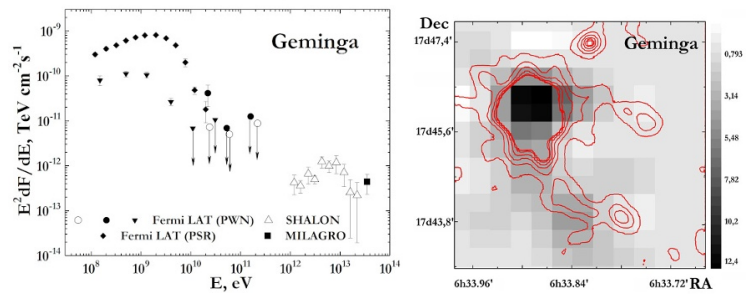

Figure 1. High energy spectral energy distribution of Geminga object.

$\triangle$ show the average spectrum from the SHALON data.

Emission maps of Geminga by SHALON (grey scale).

Red lines show Chandra data.

outer structures of PWN detected in X-rays by Chandra (Fig. 1, right). The fluxes of MeVGeV emission from Geminga PWN obtained in observations with Fermi-LAT were estimated in [8]. The $\sim 2$ degrees extended halo around Geminga was reported first by HAWC [9] and then by [10] presenting the analysis of Fermi-LAT data. Both, TeV gamma-rays and extended $\mathrm{MeV}-\mathrm{GeV}$ emission viewed by HAWC and extracted from Fermi observations could arise from the PWN associated with the Geminga SNR.

\section{$33 \mathrm{C} 58$}

$3 \mathrm{C} 58$ is a supernova remnant (SNR) classified as a PWN due to its flat radio spectrum and very bright core [11]; it is powered by the central pulsar PSR J0205 + 6449 [12]. Radio observations revealed a 6' $\times 9^{\prime}$ size filled-centre elliptical morphology of 3 C $58[13,14]$. Similar morphologies have been observed in the infrared (IR) and X-ray bands [15-17]. In X-rays 3C 58 is found to have a non-thermal spectrum from the nebula that varies with radial distance $[16,18]$ and the nebula size decreases with increasing energy [18].

Gamma-ray emission of $\mathrm{MeV}-\mathrm{GeV}$ energies from the nebula with a power-law function spectrum with a photon index of $1.61 \pm 0.21$ has been detected by Fermi-LAT [19, 20].

The source of $\mathrm{TeV} \gamma$-rays associated with the 3C 58 was detected for the first time by SHALON [21, 22] in 2011 with a statistical significance of $8.1 \sigma$. A power law can well describe the energy spectrum of $3 \mathrm{C} 58$, the photon index in the energy range $0.8-10 \mathrm{TeV}$ was derived to be $2.36 \pm 0.18$. Its overall spectral energy distribution is presented in Fig. 2 .

It is generally believed that the radio, IR and X-ray part of emission from the 3C 58 nebula is produced by the synchrotron radiation of relativistic electrons within the PWN, but the $\mathrm{GeV}$ and $\mathrm{TeV} \gamma$-rays are generated through inverse Compton scattering-off soft photon fields $[23,24]$. The spatially dependent model proposed by [25] was applied to 3C $58 \mathrm{PWN}$ to describe the observed non-thermal emission properties of 3C 58 (Figs. 2, 3).

The radio source 3C 58 was recognized as an SNR at $3.2 \mathrm{kpc}$ [26], and it coincides with the historical supernova SN 1181. But, both the dynamical age of the pulsar $5.4 \mathrm{kyr}$ determined from its spin-down rates and the age of $5.0 \pm 2.25 \mathrm{kyr}$ corresponding to the mean expansion velocity of the 3C 58 determined by comparing the VLA radio images in 1973 and
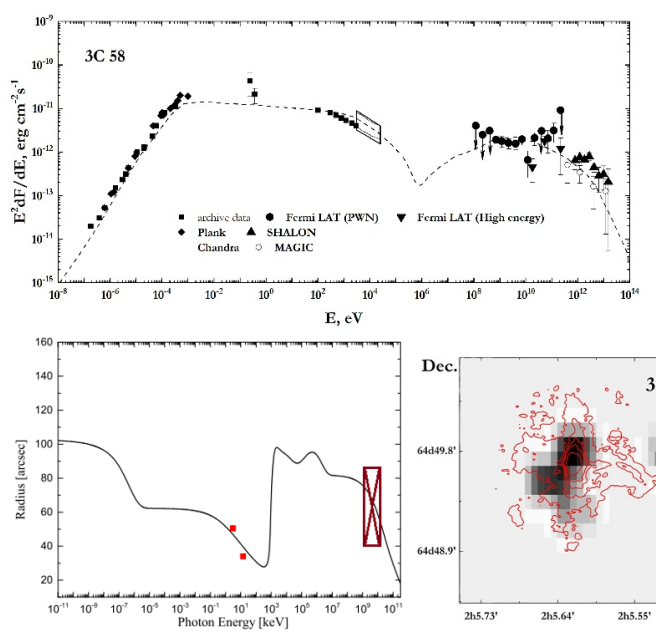

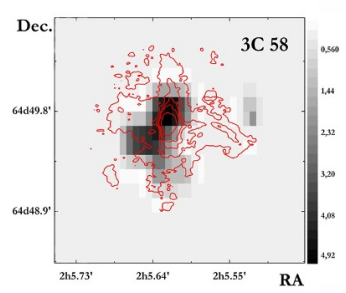

Figure 3. left: Variation of the energy-dependent radial extent of 3C 58. Line is the calculations from [25] model. The observed data at $\mathrm{TeV}$ energies is from SHALON observations. right: Emission maps of 3C 58 by SHALON (grey scale). Red lines show Chandra data. 
1998 are incompatible with the age of the remnant of SN 1181. The system age estimation from the PWN evolution and energetics gave the value of $~ 2.5 \mathrm{kyr}$ [27], also incompatible with the supernova explosion of 1181. Different studies derive ages are from 0.8 to $7 \mathrm{kyr}$.

New HI absorption study towards 3C 58 PWN 3C 58 in combination with a newly determined distance-velocity relation and by relating this PWN to a nearby H II region and SNR complex of well-known distance revealed the distance value of $2 \mathrm{kpc}$ [28], which then points to the younger age of this object. Also, approximately the type of the progenitor star of $3 \mathrm{C}$ 58 were determined in these studies that favour to a point to a late O-type star. Comparison of the result of the model (with parameters including the distance of $2 \mathrm{kpc}$ ) with the observed photon spectral energy distribution of 3C 58 is presented in Figs. 2, 3 shows modeled in [25] nebula radial extent in the radio, IR, GeV and $\mathrm{TeV}$ bands. The observed extension in $\mathrm{TeV}$ energies is consistent with this model.

\section{Conclusions}

Experimental data on $\mathrm{TeV} \gamma$-ray emission from Pulsar Wind Nebulae of the different evolution stages are obtained in the SHALON long-term observations. The extended emission at energies $>800 \mathrm{GeV}$ from Geminga was detected and compared with one from the X-ray observations with Chandra telescope. It could arise from the PWN associated with the middleaged Geminga SN remnant. The Geminga PWN structures viewed in TeV gamma-rays by SHALON and X-rays by Chandra together with the presence of extended halo detected both in MeV-GeV by Fermi-LAT and TeV by HAWC is notably different from fluxes and morphology of 3C 58 detected across the electromagnetic spectrum. Gamma-ray emission from 3C 58 PWN which may associated with historical SN 1181 was detected with SHALON in the range of $0.8-10 \mathrm{TeV}$. 3C $58 \gamma$-ray emission structure was obtained and overlaps with one visible in X-rays by Chandra. Spatially dependent particle transport model [25] was applied to $3 \mathrm{C} 58$ to compare the observed $\gamma$-ray emission spectra and radial extent at $\mathrm{TeV}$ energies with the model results. The observed extension and spectral energy distribution in $\mathrm{TeV}$ energies are consistent with this model, which points to the variation of the 3C 58 nebula extent with energy and may impact the study of the injection particle distribution and the particle acceleration within the PWN.

\section{References}

[1] M.J. Rees \& J.E. Gunn, MNRAS 167, 1 (1974)

[2] S.P. Reynolds \& R.A. Chevalier, ApJ 278, 630 (1984)

[3] M. Salvati \& B. Sacco, A\&A 485, 527 (2008)

[4] M. Salvati, A\&A 513, A28 (2010)

[5] Y.A. Shibanov et al., A\&A 448, 313 (2006)

[6] V.G. Sinitsyna, AIP (Conf. Proc.) 515, 205, 293 (2000)

[7] V.G. Sinitsyna et al., Nucl. Phys. B (Proc.Suppl.) 196, 437 (2009)

[8] M. Ackermann et al, ApJ 726, 35 (2011)

[9] R. López-Coto, F. Salesa, H. Zhou (for the HAWC collaboration), AIP (Conf. Proc.) 1792, 040014 (2017)

[10] M. Di Mauro, S. Manconi, F. Donato, Phys. Rev. D 100, 419 (2019)

[11] K.W. Weiler, N. Panagia, A\&A 70, 419 (1978)

[12] S.S. Murray, P.O. Slane, F.D. Seward et al., ApJ 568, 226 (2002)

[13] S.P. Reynolds, H.D. Aller, ApJ 327, 845 (1988)

[14] M.F. Bietenholz, ApJ 645, 1180 (2006)

[15] D.A. Green, P.A.G. Scheuer, MNRAS 258, 833 (1992)

[16] P. Slane, D.J. Helfand, E. van der Swaluw, S/S. Murray, ApJ 616, 403 (2004) 
[17] P. Slane, D.J. Helfand, S.P. Reynolds et al., ApJ 676, L33 (2008)

[18] H. An, ApJ 876, 150 (2019)

[19] A.A. Abdo et al., ApJ 699, L102 (2009)

[20] M. Ackermann et al., ApJS 209, 34 (2013)

[21] V.G. Sinitsyna \& V.Yu. Sinitsyna, J. Phys.: Conf. Ser. 409, 012110 (2013)

[22] V.G. Sinitsyna \& V.Yu. Sinitsyna, "Astroparticle, Particle, Space Physics and Detectors Physics Applications" ed S Giani, C Leroy, P-G Rancoita, L Price and R Ruchti (Singapore: World Scientific, 2014) vol 8 p 135

[23] S.J. Tanaka \& F. Takahara, MNRAS 429, 2945 (2013)

[24] F.-W. Lu, Q.-G. Gao, B.-T. Zhu, L. Zhang, MNRAS 472, 2926 (2017a)

[25] F.-W. Lu, Q.-G. Gao, B.-T. Zhu, L. Zhang, ApJ 834, 43 (2017b)

[26] D.A. Roberts, W.M. Goss, P.M. Kalberla et al., A\&A 274, 427 (1993)

[27] R.A. Chevalier, ApJ 619, 839 (2005)

[28] R. Kothes, A\&A 560, A18 (2013) 